\title{
Role of Signal Transducer and Activator of Transcription-3 in Estradiol-Mediated Neuroprotection
}

\author{
Suzan Dziennis, ${ }^{1}$ Taiping Jia, ${ }^{2}$ Oline K. Rønnekleiv, ${ }^{1,2}$ Patricia D. Hurn, ${ }^{1,2}$ and Nabil J. Alkayed ${ }^{1,2}$ \\ Departments of ${ }^{1}$ Anesthesiology and Peri-Operative Medicine and ${ }^{2}$ Physiology and Pharmacology, Oregon Health \& Science University, Portland, Oregon \\ 97239-3098
}

\begin{abstract}
Estradiol is protective in experimental cerebral ischemia, but the precise mechanisms remain unknown. Signal transducer and activator of transcription-3 (STAT3) is a transcription factor that is activated by estrogen, translocates to the nucleus, and induces the transcription of neuroprotective genes, such as $b c l-2$. We determined whether estradiol increases STAT3 activation in female rat brain after focal cerebral ischemia and whether STAT3 activation contributes to estradiol-mediated neuroprotection against ischemic brain injury. Ovariectomized (OVX) female rats with and without estradiol replacement were subjected to $2 \mathrm{~h}$ of middle cerebral artery occlusion (MCAO), and phosphorylated STAT3 (P-STAT3) and total STAT3 (T-STAT3) were quantified by Western blot analysis at 3 and $22 \mathrm{~h}$ of reperfusion. STAT3 activation was colocalized with neuronal and survival markers microtubule-associated protein 2 (MAP2) and Bcl-2 using immunohistochemistry. Infarct size was measured at $22 \mathrm{~h}$ after MCAO in estradiol-treated OVX animals in the presence and absence of STAT3 inhibitor cucurbitacin I (JSI-124) using 2,3,5-triphenyltetrazolium chloride staining. Estradiol increased P-STAT3 in the ischemic cortex cytosolic fraction at $3 \mathrm{~h}$ after MCAO without affecting T-STAT3. This was associated with increased P-STAT3 in the nuclear fraction, which remained elevated at $22 \mathrm{~h}$ after MCAO. The nuclear P-STAT3 colocalized with MAP2 and Bcl-2 within the peri-infarct zone. The P-STAT3 inhibitor JSI-124 abolished the protective effect of estradiol without affecting infarct size in untreated OVX rats. We conclude that estradiol increases STAT3 phosphorylation in neurons after MCAO and that STAT3 activation plays an important role in estradiol-mediated neuroprotection.
\end{abstract}

Key words: estrogen; STAT3; ischemia; neuroprotection; MCAO; JSI-124 (cucurbitacin I)

\section{Introduction}

We previously demonstrated that female rats sustain smaller infarcts after middle cerebral artery occlusion (MCAO) than male rats (Alkayed et al., 1998) and that chronic estrogen replacement that restores physiological levels of plasma estradiol is protective against ischemic brain injury in ovariectomized (OVX) (Rusa et al., 1999) and reproductively senescent (Alkayed et al., 2000) female rats. Estradiol exhibits acute (Bryant et al., 2005; Qiu et al., 2006) and chronic (O'Lone et al., 2004) effects. The chronic effects of estradiol are mediated via alterations in gene expression, the so-called "genomic" effects of estradiol (Xu et al., 2006). Acute, rapid effects of estradiol are believed to be mediated via so-called "nongenomic" mechanisms, such as changes in protein phosphorylation (Levin, 2002). However, the acute and chronic effects of estrogen are not mutually exclusive. Recent studies suggest that estrogen rapidly activates signal transduction pathways that ultimately lead to increased gene transcription (Bjornstrom

Received April 6, 2007; revised May 19, 2007; accepted May 28, 2007.

This work was supported by National Institutes of Health Grants F32 NS053065 (S.D.), NS044313 (N.J.A.), NS 33668 (P.D.H.), and P01 NS049210 (0.K.R., P.D.H., N.J.A.). We also acknowledge the technical assistance of Vanessa Anderson and Jennifer Young.

Correspondence should be addressed to Dr. Nabil J. Alkayed, Department of Anesthesiology and Peri-Operative Medicine, Oregon Health \& Science University, 3181 S.W. Sam Jackson Park Road, UHS-2, Portland, 0R 97239-3098. E-mail: alkayedn@ohsu.edu.

DOI:10.1523/JNEUROSCI.1558-07.2007

Copyright $\odot 2007$ Society for Neuroscience $\quad$ 0270-6474/07/277268-07\$15.00/0 and Sjoberg, 2005). Specifically, in non-neural cell lines, estrogen induces the phosphorylation of signal transducer and activator of transcription-3 (STAT3) (Bjornstrom and Sjoberg, 2002). STAT proteins reside in the cytoplasm in an unphosphorylated dormant form. In response to a wide variety of external stimuli, including cytokines, hormones and growth factors, STATs are activated by tyrosine phosphorylation (Levy and Darnell, 2002). Phosphorylated STATs then dimerize and translocate to the nucleus where they bind specific DNA regions, referred to as STATinducible elements (SIEs), to induce gene transcription. There are six known STATs: STAT1, STAT2, STAT3, STAT4, STAT5a, STAT5b, and STAT6 (Levy and Darnell, 2002). Only STAT3 has been linked to estradiol's action, neuronal survival, and tissue protection from ischemia. Specifically, STAT3 phosphorylation is increased by estradiol in an aortic endothelial cell line (Bjornstrom and Sjoberg, 2002) and mediates survival effects of neurotrophins in motor (Dolcet et al., 2001) and sensory (Alonzi et al., 2001) neurons. In heart, STAT3 promotes tissue survival after myocardial infarction (Negoro et al., 2000) and plays an important role in tolerance to ischemia acquired by ischemic preconditioning (Hattori et al., 2001; Xuan et al., 2001). Finally, STAT3 induces the expression of neuroprotective genes, such as $b c l-2$ (Linnik et al., 1995; Jia et al., 1996) and $b c l-x_{L}$ (Parsadanian et al., 1998) (Grad et al., 2000; Stephanou et al., 2000). It is not known, however, whether estradiol enhances STAT3 phosphorylation in 
brain regions affected by stroke or whether STAT3 activation contributes to estradiol's neuroprotective action in stroke.

Therefore, in the present study we tested the hypothesis that $17 \beta$-estradiol protects the brain against ischemic injury in part through STAT3 activation. We used Western blot with antiSTAT3 and anti-phospho-STAT3 antibodies to measure STAT3 expression and activation by estradiol in brain after MCAO. Furthermore, we used immunohistochemistry to determine the cell identity of phospho-STAT3-expressing cells. Finally, the STAT3 pharmacological inhibitor cucurbitacin I [JSI-124 (JSI)] (Blaskovich et al., 2003) was used to determine the role of STAT3 activation in infarct size reduction by estradiol. This is the first study to link the neuroprotective effect of estradiol against cerebral ischemia to STAT3.

\section{Materials and Methods}

This study was conducted in accordance with the National Institutes of Health guidelines for care and use of animals in research, and the protocols were approved by the Animal Care and Use Committee of Oregon Health \& Science University.

Ovariectomy and estrogen replacement. Ovariectomy was performed in rats 1-2 weeks before MCAO, and estrogen was replaced via 21-d-release pellets containing $25 \mu \mathrm{g}$ of $17 \beta$-estradiol (Innovative Research of America, Toledo, $\mathrm{OH}$ ) implanted subcutaneously on the day of ovariectomy. Plasma $17 \beta$-estradiol was measured by radioimmunoassay when the rats were killed, as described previously (Rusa et al., 1999; Alkayed et al., 2000).

Experimental stroke in rat. Focal cerebral ischemia was induced in rats using the intraluminal MCAO model, as described previously (Alkayed et al., 1998; Rusa et al., 1999). Briefly, ovariectomized female rats (10-12 weeks of age, $200-250 \mathrm{~g}$ body weight) with and without estradiol replacement were anesthetized with halothane $\left(1 \%\right.$ in $\mathrm{O}_{2}$-enriched air via face mask) and instrumented with a femoral catheter for monitoring arterial blood pressure and gases. Brain tissue perfusion was monitored over the ipsilateral parietal cortex using a laser-Doppler probe (Moor Instruments, Oxford, UK) placed $6 \mathrm{~mm}$ lateral and $2 \mathrm{~mm}$ posterior to bregma to assess vascular occlusion and reperfusion. Head and body temperatures were controlled and monitored through a temporalis muscle and rectal thermoprobes. Vascular occlusion was achieved by advancing a 4-0 nylon filament with heat-rounded tip into the internal carotid artery until a drop was observed in laser-Doppler perfusion (LDP). LDP was continuously monitored during the occlusion period and over $30 \mathrm{~min}$ after reperfusion. Only animals with a $>50 \%$ drop in LDP relative to preocclusion baseline were used in this study. Vascular occlusion was maintained for $2 \mathrm{~h}$, and then the occluding filament was withdrawn to permit reperfusion, and the rat was recovered for either 3 or $22 \mathrm{~h}$. Each animal was deeply anesthetized at the appropriate time point with halothane, blood samples were obtained by cardiac puncture for measurement of plasma $17 \beta$-estradiol, and the brains were collected and processed for subcellular fractionation, immunohistochemistry, or determination of infarct volume. To quantify infarct, the brain was harvested at $22 \mathrm{~h}$ of reperfusion, 2-mm-thick coronal brain sections (seven sections per brain) were stained with 2,3,5-triphenyltetrazolium chloride and fixed in $10 \%$ formalin, and infarcted areas were measured in digital images of brain slices using MCID image analysis software (InterFocus Imaging, Linton, UK). Infarct volume was calculated by integrating infarcted areas across the rostral-caudal axis and expressed as a percentage of the contralateral hemisphere to account for edema.

The STAT3 inhibitor JSI-124 was initially given intraperitoneally at a dose of $1 \mathrm{mg} / \mathrm{kg}$ at $15 \mathrm{~min}$ before MCAO. However, we observed that JSI induces slight fluctuations in arterial blood pressure during MCAO. To rule out an effect of these fluctuations on ischemic severity and infarct size, we also administered JSI at 15 min after MCAO.

Subcellular fractionation. Cortical tissue from the area shaded in Figure 3 (peri-ischemia; between +2 and $-3 \mathrm{~mm}$ from bregma) was dissected at 3 and $22 \mathrm{~h}$ of reperfusion and flash frozen in 2-methyl butane over dry ice. Tissue was stored at $-80^{\circ} \mathrm{C}$ until use. Nuclear and cytosolic fractions were prepared as described previously (Sonnenberg et al., 1989; Takeuchi et al., 1993) with the following modifications. Cortical tissue (80-100 $\mathrm{mg}$ ) was homogenized at low speed in 400-500 $\mu \mathrm{l}$ of ice-cold hypotonic lysis buffer containing Roche (Mannheim, Germany) complete Mini EDTA-free protease inhibitor, phosphatase inhibitor cocktails I and 2 (Sigma, St. Louis, MO), 1 mm PMSF, and 1 mм DTT. Nuclear fractions were dialyzed using Slide-A-Lyzer Mini dialysis units (Pierce, Rockford, IL). Protein concentrations were measured using the Pierce BCA kit. The purity of each fraction was evaluated by probing cytosolic and nuclear fractions with antibodies against the cytosolic protein AKT and the nuclear protein Histone-3. Both antibodies were obtained from Cell Signaling Technology (Danvers, MA).

Western blot. Phosphorylated (Tyr 705; s.c.-7993-R; P-STAT3) and total (s.c. H-190; T-STAT3) STAT3 antibodies were purchased from Santa Cruz Biotechnology (Santa Cruz, CA). As a positive control for STAT3 activation, we used interferon- $\alpha$-treated HeLa cell extracts (Cell Signaling Technology). Protein samples $(50 \mu \mathrm{g})$ from nuclear or cytosolic fractions were boiled for $5 \mathrm{~min}$ in $1 \times$ sample buffer (Invitrogen, Carlsbad, CA) before loading onto a Criterion $4-15 \%$ Tris-HCl gel (BioRad, Hercules, CA). Gels were transferred using the Criterion transfer system at $100 \mathrm{~V}$ for $45 \mathrm{~min}$ onto nitrocellulose membranes (Bio-Rad). Membranes were blocked in PBST (10 mM sodium phosphate, $150 \mathrm{~mm}$ sodium chloride, $\mathrm{pH} 7.4$, and $0.1 \%$ Tween 20 ) with either $5 \%$ bovine serum albumin (BSA; P-STAT3) or 5\% milk (T-STAT3) for $1 \mathrm{~h}$. Membranes were incubated with the appropriate blocking solution containing the antibody at 1:1000 dilution overnight at $4^{\circ} \mathrm{C}$. After washing, membranes were incubated for $1 \mathrm{~h}$ at room temperature with horseradish peroxidase-conjugated secondary antibodies (Bio-Rad) diluted 1:5000 in corresponding blocking buffer. P-STAT3 blots were stripped for $1 \mathrm{~h}$ at room temperature in $62.5 \mathrm{~mm}$ Tris, $\mathrm{pH} 6.8,2 \%$ SDS, and $0.7 \%(\mathrm{v} / \mathrm{v})$ $\beta$-mercaptoethanol, followed by extensive washing in PBST before blocking and incubation with anti-STAT3 antibody. Immunoreactive bands were visualized using Super Signal Dura (Pierce) chemiluminescence. Band intensity was recorded and analyzed using the Kodak (Rochester, NY) Image Station and software (2000R). Gels were stained with Coomassie blue, photographed with a CoolSNAPcf digital camera (Roper Scientific, Tucson, AZ), and analyzed with MCID image analysis software (InterFocus Imaging). The P-STAT3 and T-STAT3 band densities were normalized to total protein load obtained from the Coomassie-stained gel.

Immunohistochemistry. Brains were harvested at $22 \mathrm{~h}$, cut into $2 \mathrm{~mm}$ coronal sections, fixed with $4 \%$ paraformaldehyde for $4-5 \mathrm{~h}$ with gentle shaking at $4^{\circ} \mathrm{C}$, and cryopreserved in $20 \%$ sucrose overnight at $4^{\circ} \mathrm{C}$. Tissue blocks from ischemic $(n=4)$ or sham-operated $(n=2)$ animals were frozen in 2 -methylbutane at $-55^{\circ} \mathrm{C}$, sectioned on a cryostat at 20 $\mu \mathrm{m}$, and thaw mounted on Superfrost Plus glass slides (Fisher Scientific, Pittsburgh, PA). The sections were stored at $-20^{\circ} \mathrm{C}$ until used. Sections were incubated at $4^{\circ} \mathrm{C}$ overnight with the following primary antisera: T-STAT3 (rabbit polyclonal s.c.-482; 1:2000; Santa Cruz Biotechnology), P-STAT3 (rabbit polyclonal 9131 Tyr 705; 1:1000-1:3000; Cell Signaling Technology), Bcl-2 (monoclonal M 0887; 1:100; Dako North America, Carpinteria, CA), microtubule-associated protein 2 (MAP2; chicken polyclonal CPCA-MAP2; 1:10,000; Encor Biotechnology, Alachua, FL) and Cy3-conjugated glial fibrillary acidic protein (GFAP) (monoclonal C 9205; 1:10,000-1:20,000; Sigma). Some sections were incubated with rabbit polyclonal antiserum to T-STAT3 or P-STAT3 that had been preadsorbed with the immunogen peptide [s.c.-482p (Santa Cruz Biotechnology) and 1195 (Cell Signaling Technology) for T-STAT3 and P-STAT3, respectively] at $10 \mu \mathrm{g} / \mathrm{ml}$ before application to the tissue section. The following day, sections were washed in PBS (0.1 M phosphate buffer, $\mathrm{pH} 7.4$, and $0.15 \mathrm{M} \mathrm{NaCl}$ ) for $30 \mathrm{~min}$, pretreated with $5 \%$ normal goat serum for $1 \mathrm{~h}$, and then incubated for $2 \mathrm{~h}$ at room temperature in biotinylated IgG [donkey anti-rabbit, 1:300; anti-mouse, 1:300 (Jackson ImmunoResearch); or anti-chicken (Vector Laboratories, Burlingame, CA), $5 \mu \mathrm{g} / \mathrm{ml}$ ]. After rinsing in PBS, the sections were incubated for $1 \mathrm{~h}$ at room temperature in streptavidin-conjugated Cy3 (1:1000), rinsed for $3-6 \mathrm{~h}$ in PBS, and protected with a coverslip applied using a glycerolglycine buffer $(2: 1 ; \mathrm{pH} 8.6)$ containing $5 \%$ n-propyl gallate to reduce photobleaching (Giloh and Sedat, 1982). Both the primary and second- 
Table 1. Physiological variables during middle cerebral artery occlusion

\begin{tabular}{|c|c|c|c|c|c|c|c|c|}
\hline Group $(n)$ & $\begin{array}{l}\mathrm{MAP}^{a} \\
(\mathrm{mmHg})\end{array}$ & $\begin{array}{l}\text { Arterial blood } \\
\mathrm{pH}\end{array}$ & $\begin{array}{l}\mathrm{PaCO}_{2}^{b} \\
(\mathrm{mmHg})\end{array}$ & $\begin{array}{l}\mathrm{PaO}_{2}{ }^{\mathrm{C}} \\
(\mathrm{mmHg})\end{array}$ & $\begin{array}{l}\text { Rectal } \\
\text { Temp. }\left({ }^{\circ} \mathrm{C}\right)\end{array}$ & $\begin{array}{l}\text { Head Temp. } \\
\left({ }^{\circ} \mathrm{C}\right)\end{array}$ & $\begin{array}{l}\text { Blood glucose } \\
(\mathrm{g} / \mathrm{dl})\end{array}$ & $\mathrm{LDF}^{d}$ \\
\hline OVX plus vehicle (11) & $99 \pm 3$ & $7.43 \pm 0.01$ & $45 \pm 1$ & $132 \pm 4$ & $36.9 \pm 0.1$ & $37.1 \pm 0.1$ & $121 \pm 6$ & $37 \pm 2$ \\
\hline EST plus vehicle (8) & $105 \pm 3$ & $7.47 \pm 0.01$ & $40 \pm 2$ & $120 \pm 4$ & $37.0 \pm 0.1$ & $36.8 \pm 0.1$ & $119 \pm 7$ & $40 \pm 2$ \\
\hline OVX plus JSI-124 (8) & $93 \pm 3$ & $7.45 \pm 0.01$ & $45 \pm 2$ & $139 \pm 3^{*}$ & $36.8 \pm 0.1$ & $36.9 \pm 0.1$ & $126 \pm 8$ & $34 \pm 4$ \\
\hline EST plus JSI-124 (11) & $100 \pm 3$ & $7.43 \pm 0.01$ & $43 \pm 2$ & $129 \pm 3$ & $37.0 \pm 0.1$ & $37.0 \pm 0.1$ & $117 \pm 6$ & $30 \pm 3$ \\
\hline
\end{tabular}

Values are mean \pm SEM. EST, Estrogen-replaced; $n$, number of animals; Temp., temperature. ${ }^{*} p<0.05$, ANOVA.

${ }^{a}$ Mean arterial blood pressure.

${ }^{b}$ Arterial $\mathrm{CO}_{2}$ tension.

'Arterial $\mathrm{O}_{2}$ tension.

daser-Doppler flowmetry.

ary antisera were diluted in Tris-(hydroxymethyl)aminomethane $(0.5 \%$; Sigma) in phosphate buffer containing $0.7 \%$ seaweed gelatin (Sigma), $0.4 \%$ Triton X-100 (Sigma), and 3\% BSA (Sigma), adjusted to pH 7.6. For P-STAT3 staining, or colocalization of P-STAT3 with Bcl-2, MAP2, or GFAP, the procedure developed by Munzberg et al. (2003) was used with slight modification. The tissue was pretreated in $1 \% \mathrm{NaOH}$ and $0.5-1 \% \mathrm{H}_{2} \mathrm{O}_{2}$ in $\mathrm{H}_{2} \mathrm{O}$ for $20 \mathrm{~min}, 0.3 \%$ glycine for $10 \mathrm{~min}$, and $0.03 \%$ SDS for $10 \mathrm{~min}$. Thereafter, the sections were blocked for $1 \mathrm{~h}$ with $5 \%$ normal goat serum in PBS/0.25\% Triton X-100. The different antisera were diluted in the same blocking solution before being applied to the tissue sections overnight. Slides were analyzed and photographed using a Nikon (Tokyo, Japan) E800 microscope equipped with epifluorescence and a Nikon DS digital camera. Slides were also analyzed using confocal microscopy with a Leica (Bannockburn, IL) TCS SP confocal system using a $40 \times$ numerical aperture 1.25 PL APO objective. Individual sections, $0.488 \mu \mathrm{m}$ apart, were imaged by sequential excitation with the 488 $\mathrm{nm}$ line of an Ar gas laser and the $561 \mathrm{~nm}$ line of a DPSS laser and projected into one plane.

Statistical analysis. Physiological variables during MCAO and differences in infarct size were analyzed with one-way ANOVA and post hoc Newman-Keuls test. Densitometric measurements of P-STAT3 and T-STAT3 Western blots were evaluated separately with Student's $t$ test, as were the differences in serum hormone levels. The criterion for statistical significance was $p<0.05$. All values are reported as mean \pm SEM.

\section{Results}

Physiological variables during MCAO were maintained within a physiological range (Table 1$)$. At the time that the rats were killed, plasma $17 \beta$-estradiol was $70 \pm 6 \mathrm{pg} / \mathrm{ml}$ in estradiol-replaced compared with $14 \pm 1 \mathrm{pg} / \mathrm{ml}$ in untreated ovariectomized rats $(n=18$ per group; $p<0.001)$. Mean laser-Doppler cortical perfusion during MCAO was similar among all experimental groups (Table 1).

\section{Estradiol increases cytosolic STAT3 phosphorylation in cortex after MCAO}

We first measured STAT3 phosphorylation in the cytosolic fraction prepared from the cerebral cortex at $3 \mathrm{~h}$ after MCAO. STAT3 phosphorylation was barely detectable in the cytosolic fractions from the contralateral side and sham-operated animals (data not shown). However, STAT3 phosphorylation was induced after $\mathrm{MCAO}$ in the cerebral cortex cytosolic fraction. Figure 1 also shows that estrogen replacement further enhanced STAT3 phosphorylation in postischemic cytosolic fractions compared with unreplaced OVX animals $(n=5$ per group; $p<0.05)$. Estradiol did not change T-STAT3 protein at this early time point, suggesting that estradiol enhances the activation of STAT3 but does not alter the amount of STAT3 protein in the cerebral cortex after MCAO. Interestingly, STAT3 phosphorylation was not different between estradiol-treated and untreated groups at $22 \mathrm{~h}$ of reperfusion ( $n=5$ per group; data not shown), suggesting that STAT3 activation in the cytosol is transient.

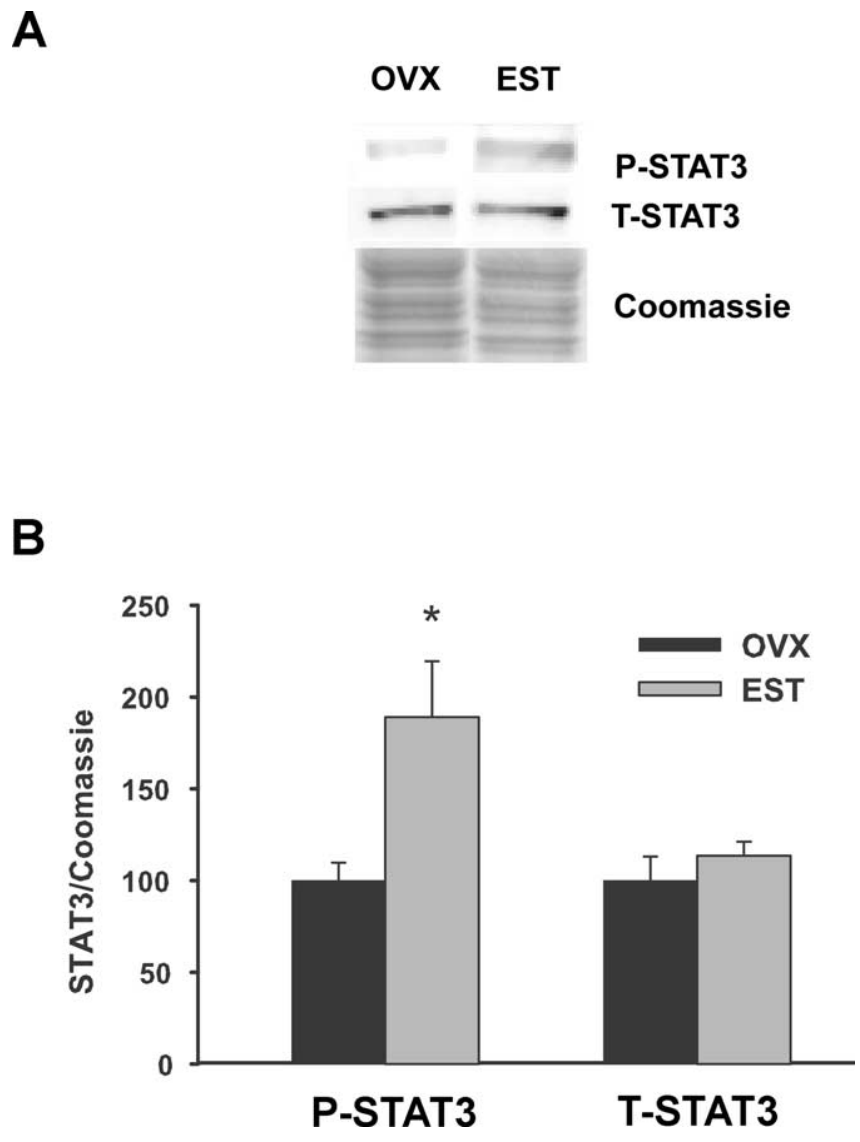

Figure 1. Estradiol replacement increases cytosolic STAT3 phosphorylation but not total STAT3 in the cerebral cortex after MCA0. Cytosolic extracts were prepared from OVX and estradiol-replaced (EST) female rat brains $3 \mathrm{~h}$ after MCAO, and Western blot was performed with an antibody against P-STAT3 or T-STAT3. A, Representative P-STAT3 and T-STAT3 blots and Coomassie-stained gel showing equal protein loading. $\boldsymbol{B}$, Summary graph depicting the changes in cytosolic STAT3 relative to total protein load. All values were normalized to mean cytosolic P-STAT3 and T-STAT3 in ovariectomized animals, which were set at 100\%. ${ }^{*} p<0.05$ for the effect of EST on cytosolic P-STAT3 ( $n=5$ per group).

\section{Estradiol increases nuclear STAT3 phosphorylation in cortex after MCAO}

Because STAT3 is translocated to the nucleus after activation, we then determined whether estradiol increases nuclear STAT3 phosphorylation. As in the cytosolic fractions, very little P-STAT3 was observed in nuclear fractions from the contralateral side. However, in agreement with the effect of estradiol on the cytosolic fractions, estradiol increased ipsilateral nuclear P-STAT3 at $3 \mathrm{~h}$ after MCAO (data not shown). In contrast to the cytosolic fraction, nuclear P-STAT3 was also increased at $22 \mathrm{~h}$ after MCAO (Fig. 2) $(n=5 ; p<0.05)$, suggesting that estradiol 
A

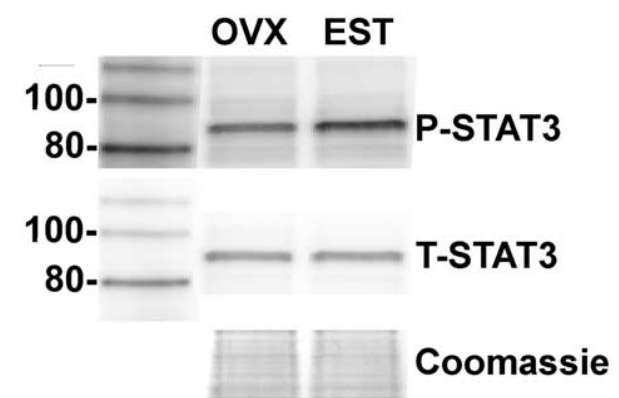

B

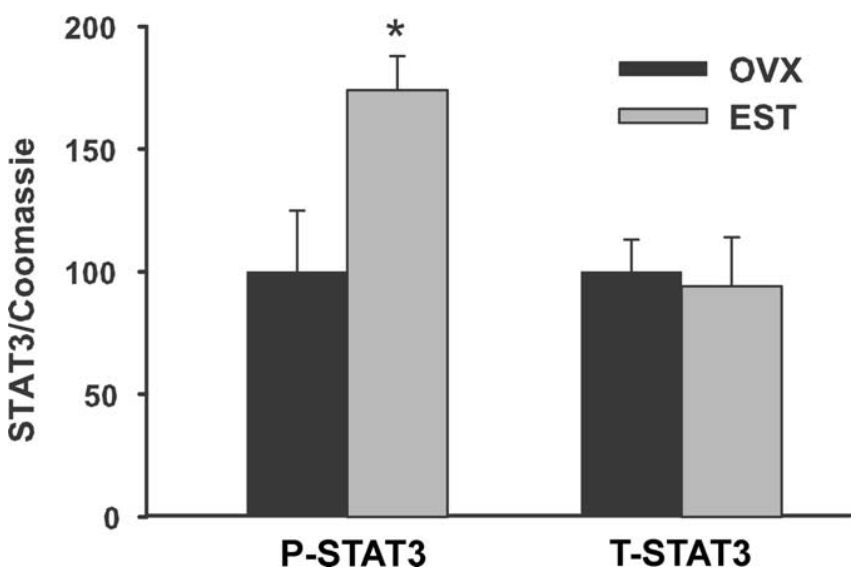

Figure 2. Estradiol replacement increases nuclear P-STAT3 but not T-STAT3 after MCAO. Nuclear extracts were prepared from the cerebral cortex of OVX and estradiol-replaced (EST) female rat brains at $22 \mathrm{~h}$ after $\mathrm{MCA} 0$, and Western blot was performed with an antibody against P-STAT3 or T-STAT3. A, Representative P-STAT3 and T-STAT3 blots and Coomassie-stained gel. $\boldsymbol{B}$, Summary graph depicting the changes in nuclear STAT3 relative to total protein load. All values were normalized to mean nuclear P-STAT3 and T-STAT3 in ovariectomized animals, which were set at $100 \%$. ${ }^{*} p<0.05$ for the effect of EST on nuclear P-STAT3; $n=5$ per group for P-STAT $; n=4$ for T-STAT3.

induces early translocation of STAT3 to the nucleus and that STAT3 remains activated in the nucleus for $24 \mathrm{~h}$ after MCAO. In accordance with increased protein translocation, T-STAT3 was also increased in the nuclear fraction at $3 \mathrm{~h}$, although this difference was not statistically significant $(p=0.09 ; n=5)$ and disappeared at $22 \mathrm{~h}$ after $\mathrm{MCAO}(n=4)$.

\section{STAT3 activation in ischemic brain colocalizes with neuronal} and survival markers

To determine cell type and subcellular localization of P-STAT3, double immunofluorescence labeling was performed in estradiol-replaced animals with an anti-P-STAT3 antibody and cell type-specific antibodies MAP2 (neuronal marker) (Fig. 3B) and GFAP (astrocytic marker; data not shown). In agreement with Western blot analysis, P-STAT3 was detected at very low levels in the contralateral side (Fig. $3 B$ ) (representative of $n=4$ ) and sham-operated animals ( $n=2$; data not shown). In the ischemic core, no MAP2 or P-STAT3 immunofluorescence was detected, indicating widespread cell death. In contrast, in the peri-ischemic region, strong P-STAT3 immunofluorescence was observed in numerous nuclei, which predominantly colocalized with MAP2 (Fig. 3B) (representative of $n=4$ ), although a few P-STAT3-expressing cells were also GFAP positive (data not shown), suggesting that within $24 \mathrm{~h}$ after MCAO, STAT3 is selec- tively activated in peri-infarct neurons. Sections incubated with rabbit polyclonal antiserum to P-STAT3 that had been preabsorbed with the immunogen showed no immunoreactivity, confirming the specificity of the anti-phospho-STAT3 antibody. We then examined whether cells expressing P-STAT3 also express Bcl-2 (Fig. 3C). In the ischemic core, no Bcl-2 or P-STAT3 immunofluorescence was detected. Neurons in the peri-ischemic region were immunoreactive for both $\mathrm{P}-\mathrm{STAT} 3$ and $\mathrm{Bcl}-2(n=4$ independent observations), suggesting that P-STAT3 and $\mathrm{Bcl}-2$ expression colocalize in the same cells.

\section{STAT3 inhibition abolishes protection by estradiol}

To determine whether estradiol-induced STAT3 phosphorylation contributes to the neuroprotective effect of estradiol, we evaluated the effect of the P-STAT3 inhibitor JSI-124 on infarct size after MCAO in estradiol-treated and untreated OVX rats. STAT3 phosphorylation was significantly reduced by JSI-124 to $57 \pm 10.5 \%(n=6)$ after ischemia (Fig. 4) relative to vehicle $(100 \pm 15.2 \% ; 40 \%$ DMSO in water; $n=4 ; p<0.05)$, suggesting that the inhibitor crosses the blood-brain barrier and effectively inhibits STAT3 phosphorylation in brain after MCAO. More importantly, JSI significantly increased infarct size at $22 \mathrm{~h}$ after MCAO in estradiol-treated rats $(n=8$ in vehicle and $n=11 \mathrm{JSI}$; $p<0.05)$. Interestingly, JSI- 124 had no effect on infarct size in untreated ovariectomized rats ( $n=11$ vehicle; $n=8$ JSI), consistent with the lower level of STAT3 phosphorylation in OVX rats. Because there were no differences in infarct size between the animals treated with JSI before $(n=4)$ or after $(n=7)$ MCAO, animals from the two groups were combined for this analysis (Fig. 5).

\section{Discussion}

The results of this study show that (1) estradiol increases STAT3 phosphorylation in cytosolic and nuclear fractions after MCAO in ovariectomized female rats, (2) STAT3 activation is localized in neurons and cells expressing Bcl-2 within the peri-ischemic region, and (3) STAT3 inhibition abolishes the reduction in infarct size by estradiol after MCAO. We conclude that STAT3 is an important mechanism used by estradiol to protect neurons after cerebral ischemia.

Estradiol is protective against ischemic brain damage via multiple mechanisms of actions (Suzuki et al., 2006), which include rapid, "nongenomic" effects and chronic, "genomic" effects (Bryant et al., 2006; Suzuki et al., 2006). In the classical view of estrogen signaling, the hormone binds its receptor [Fig. 6, estrogen receptor (ER)], which acts as a transcription factor that binds to a known consensus sequence referred to as estrogen response element (ERE) to induce gene transcription. It is now recognized that estradiol exhibits other effects, the so called "rapid" or nongenomic effects that may eventually affect gene expression, particularly if the target of estradiol's acute effect is a transcription factor such as CREB (cAMP response element-binding protein) (Wade and Dorsa, 2003) or STAT3 (Bjornstrom and Sjoberg, 2002).

STAT proteins are transcription factors that reside in the cytoplasm in an unphosphorylated dormant form. When activated by phosphorylation, STATs dimerize and translocate to the nucleus, where they bind specific DNA regions to induce gene transcription (Levy and Darnell, 2002). Based on previous reports linking estrogen to STAT3 activation in vitro (Bjornstrom and Sjoberg, 2002) and the role of STAT3 in neuronal survival (Alonzi et al., 2001; Schweizer et al., 2002), we here tested the hypothesis that estradiol increases STAT3 phosphorylation after ischemia in 
A

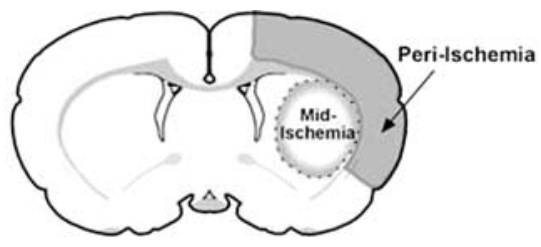

B
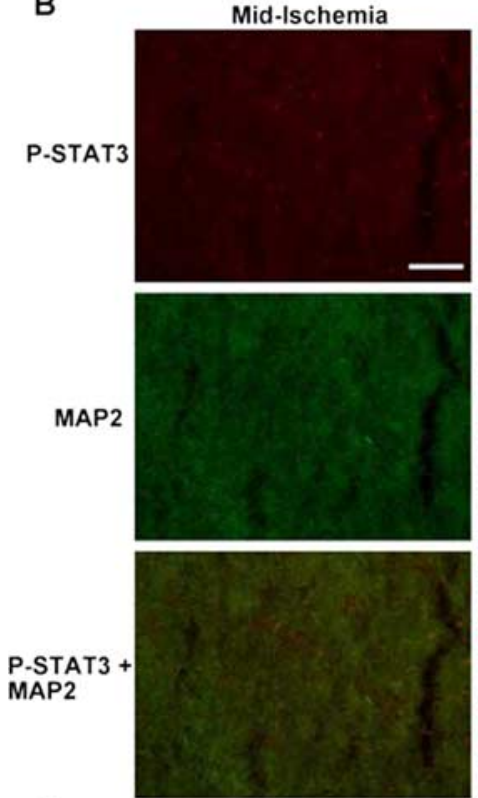

C

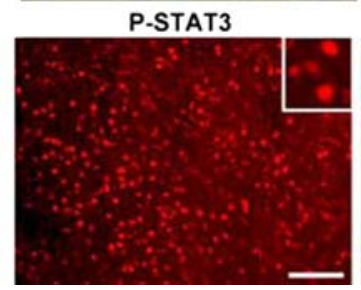

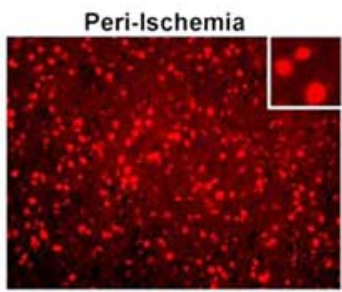
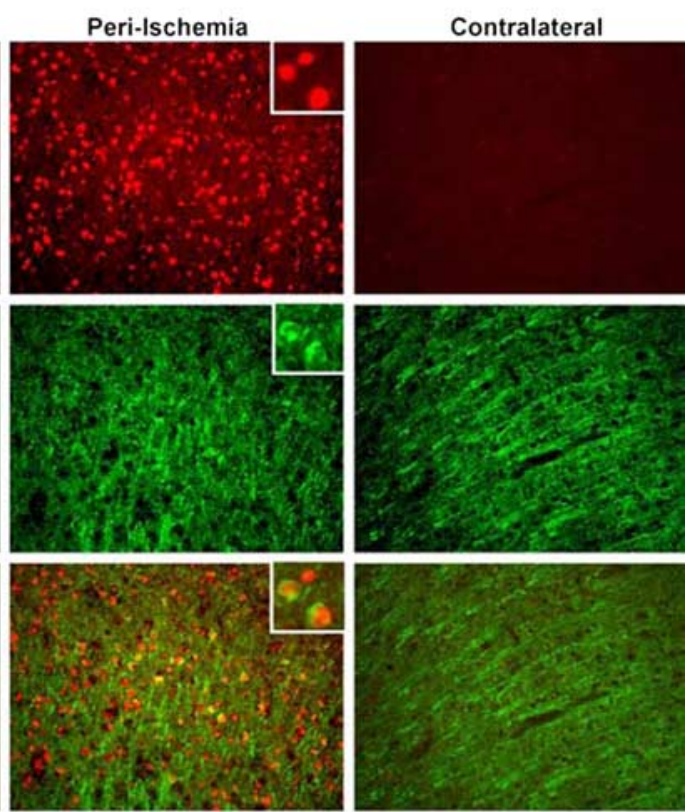

P-STAT3 + Bcl-2

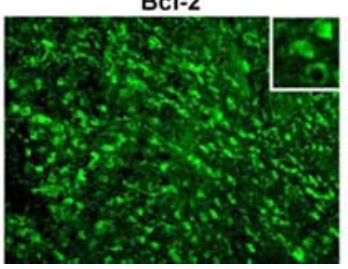

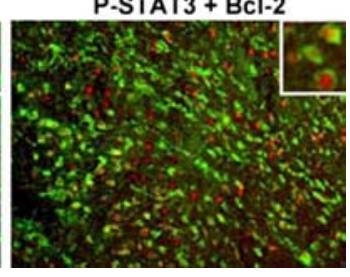

Figure 3. Colocalization of P-STAT3, MAP2, and BCl-2 expression in the peri-ischemic cerebral cortex at $22 \mathrm{~h}$ after MCAO in estradiolreplaced OVX rats. $A$, Schematic drawing of a coronal rat brain slice illustrating the areas used for cortical dissections and immunohistochemical analysis (shaded area). The same designated areas were used for the contralateral side. B, Photomicrographs from midischemic (striatum) and peri-ischemic (cortex) regions on the ipsilateral and contralateral sides. The neuronal marker MAP2 (green) was highly expressed in most brain regions, including the peri-ischemic cortex and the contralateral cortex, but not in mid-ischemic tissue. P-STAT3 (red) was densely expressed in the peri-ischemic area only, which colocalized with MAP2. C, P-STAT3 (red) and Bcl-2 (green) exhibited a similar distribution pattern and colocalized in most peri-ischemic cortical cells. Insets illustrate higher-power examples of the immunoreactivity. Images are representative of four immunohistochemical runs from four different animals. Scale bars, $100 \mu \mathrm{m}$.

vivo and that STAT3 activation contributes to estrogen-mediated neuroprotection. Our data demonstrate that STAT3 is activated in the cytoplasm as early as $3 \mathrm{~h}$ after MCAO and that estradiol significantly increases the level of phosphoSTAT3 in cytosolic fractions without affecting total STAT3. To determine whether activated STAT3 in the cytosol translocates to the nucleus, we then measured phosphorylated and total STAT3 in the nuclear fractions. We found that estradiol also increases the level of activated STAT3 in the nuclear fractions. The increase in cytosolic STAT3 phosphorylation by estradiol was transient, because there was no difference between estradioltreated and untreated groups at $22 \mathrm{~h}$ of reperfusion. However, the increase in nuclear STAT3 phosphorylation by estradiol was sustained for up to $22 \mathrm{~h}$. These observations suggest that STAT3 is rapidly activated by ischemic stress in the cytosol, followed shortly by translocation to the nucleus, where activated STAT3 remains for at least $24 \mathrm{~h}$, presumably to sustain long-term gene expression. As illustrated in Figure 6, which summarizes our working model of how estradiol uses STAT3 as a mediator of neuroprotection after ischemia, STAT3 phosphorylation is increased in the cytosol in response to estradiol. STAT3 then translocates to the nucleus, where it binds to consensus DNA sequences in neuroprotective genes such as $b c l-2$.

To determine whether STAT3 is activated specifically in neurons and whether STAT3 activation is associated with neuroprotective gene expression, we determined whether P-STAT3 immunoreactivity colocalized with MAP2 and Bcl-2. P-STAT3 colocalized with MAP2 immunoreactivity, suggesting that STAT3 is activated after MCAO in neurons. Furthermore,

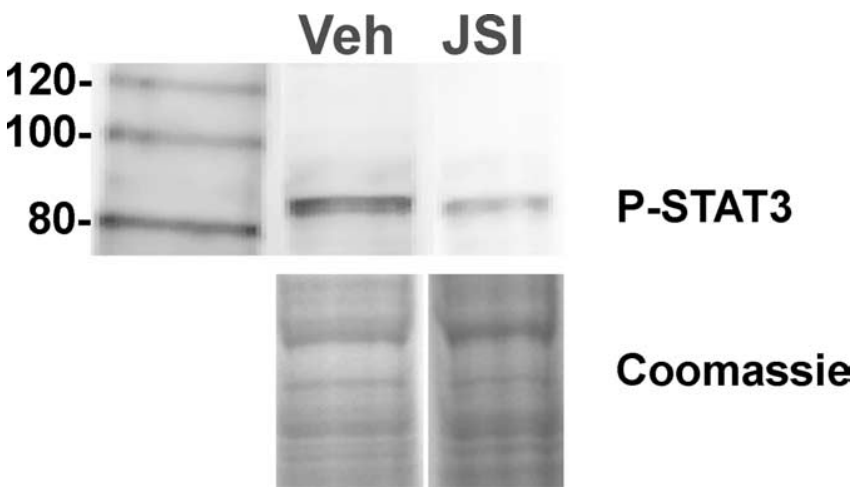

Figure 4. JSI-124 inhibits nuclear STAT3 phosphorylation in brain after systemic administration. Shown is Western blot analysis of P-STAT3 at $3 \mathrm{~h}$ after MCAO in estradiol-replaced OVX rats, which were injected with $1 \mathrm{mg} / \mathrm{kg} J \mathrm{JI}-124$ intraperitoneally $15 \mathrm{~min}$ before MCAO. Western blotting was performed on ipsilateral cortical tissue using an antibody against P-STAT3. Representative P-STAT3 blot with Coomassie-stained gel. Veh, Vehicle.
P-STAT3 colocalized with Bcl-2 within the peri-infarct region, consistent with reports suggesting $b c l-2$ regulation by STAT3. Because STAT3 is activated in brain under stress conditions, such as ischemia, and estradiol further increases levels of P-STAT3, estradiol may preferentially use STAT3 as an alternative pathway to stimulate gene transcription after MCAO.

To determine the role of STAT3 activation in estradiolmediated neuroprotection, we combined estradiol replacement with the STAT3 inhibitor JSI-124. We demonstrated that JSI-124 reduces STAT3 phosphorylation in brain after systemic administration, suggesting that JSI- 124 crosses the blood-brain barrier at sufficient concentrations to effectively inhibit STAT3 activation in brain. Using JSI-124, we then demonstrated that STAT3 inhibition abolishes the ability of estradiol to protect against ischemic brain injury, suggesting that STAT3 is an important player in the mechanism of neuroprotection by estradiol. JSI-124 did not alter infarct size in untreated ovariectomized female rats, suggesting that the effect of JSI-124 observed in our study was specific to 


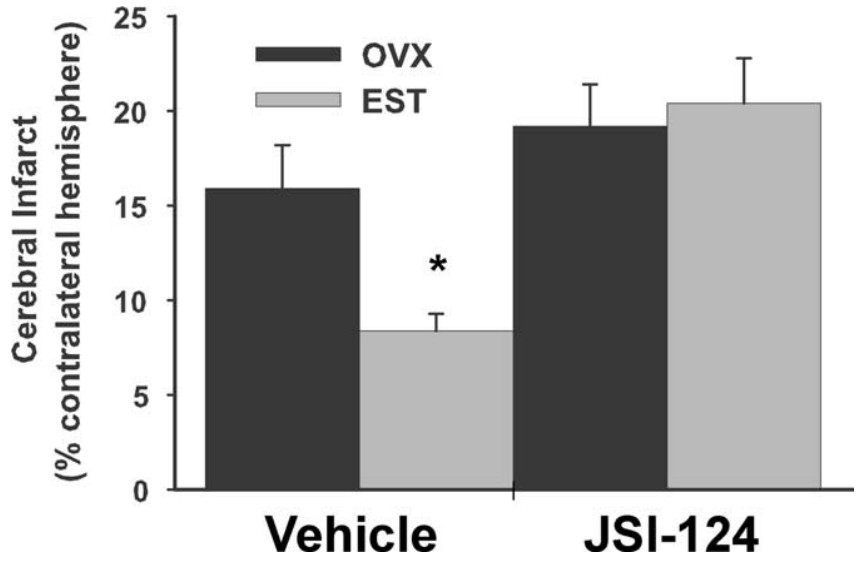

Figure 5. STAT3 inhibition abolishes the protective effect of estradiol. Infarct size was measured at $22 \mathrm{~h}$ after MCAO in estradiol-replaced (EST) and untreated OVX rats with and without the STAT3 inhibitor JSI-124 (1 mg/kg). The inhibitor was administered $15 \mathrm{~min}$ before or after MCA0. Because there were no differences, infarct size in the two groups was combined in this figure. Infarct size was significantly smaller in vehicle-treated EST than in the corresponding OVX group. However, the difference disappeared in the presence of JSI. $n=11$ OVX, $n=8 \mathrm{EST}$, $n=80 \mathrm{VXX}$ plus JSI- 124 , and $n=11 \mathrm{EST}$ plus JSI- 124 animals. ${ }^{*} p<0.5$ compared with other groups.

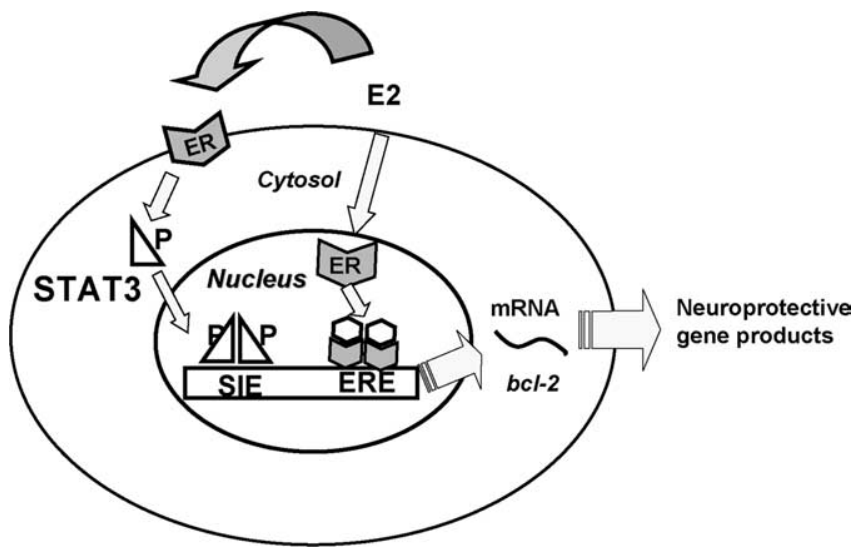

Figure 6. Working model for estradiol (E2)-mediated neuroprotection via STAT3. Estrogen induces STAT3 phosphorylation in the cytosol after cerebral ischemia. Activated STAT3 translocates to the nucleus and induces neuroprotective genes, such as bcl-2. Neuroprotection through estradiol-induced STAT3 phosphorylation may occur in parallel to or independent of the classical ER-mediated transcription via ERE. The SIE is the STAT3 consensus binding sequence.

estradiol-induced neuroprotection. JSI-124 has previously been demonstrated to selectively inhibit STAT3 phosphorylation and DNA binding without affecting other kinases involved in survival pathways such as AKT, ERK1/2 (extracellular signal-regulated protein kinase 1/2), JNK (c-Jun N-terminal protein kinase), and Src (Blaskovich et al., 2003). Furthermore, JSI-124 inhibits STAT3-dependent cancer cell proliferation both in vitro and in vivo without apparent toxicity (Blaskovich et al., 2003; Nefedova et al., 2005). In our study, JSI-124 caused fluctuations in arterial blood pressure when administered before MCAO. These fluctuations, however, disappeared when JSI-124 was administered after MCAO.

The significance of STAT3 to neuronal survival is highlighted by the observation that STAT3 gene deletion is lethal (Akira, 2000) and that neuronal survival is impaired after nerve lesion in neuron-specific STAT3-null mice (Schweizer et al., 2002). The role of STAT3 in neuronal survival is further supported by the observation that STAT3 is activated in brain after ischemic injury
(Justicia et al., 2000; Suzuki et al., 2001) and that multiple growth factors and cytokines use the STAT3 pathway to promote neuronal survival. For example, erythropoietin (EPO) protects the brain against ischemia-induced apoptosis (Ehrenreich et al., 2002; Solaroglu et al., 2003) and promotes regeneration of CNS neurons via STAT3 (Kretz et al., 2005). EPO also maintains Bcl-2, which correlates with cell survival after hypoxia/ischemia (Kumral et al., 2006). G-CSF (granulocyte colony stimulating factor) also protects the brain after an ischemic event (Solaroglu et al., 2006a). This protection correlates with increased phosphoSTAT3 and increased Bcl-2 (Komine-Kobayashi et al., 2006; Solaroglu et al., 2006b) in surviving cells. Finally, blockade of IL-6 (interleukin-6) signaling reduces STAT3 phosphorylation in the peri-infarct area of the cortex, which is linked to increased apoptosis and infarct size (Yamashita et al., 2005). In contrast to our finding, a single report (Satriotomo et al., 2006) found that inhibiting STAT3 reduced brain damage after MCAO. In their study, total STAT3 expression was reduced by small interfering RNA, which reduces both P-STAT and T-STAT, compared with our study, in which STAT3 phosphorylation was reduced by JSI124 , but total protein was not changed. Furthermore, Satriotomo et al. (2006) showed that microglia/macrophages were the predominant cell type expressing P-STAT3 after ischemia, suggesting that STAT3 may play differential roles in different cell types.

\section{References}

Akira S (2000) Roles of STAT3 defined by tissue-specific gene targeting. Oncogene 19:2607-2611.

Alkayed NJ, Harukuni I, Kimes AS, London ED, Traystman RJ, Hurn PD (1998) Gender-linked brain injury in experimental stroke. Stroke 29:159-165.

Alkayed NJ, Murphy SJ, Traystman RJ, Hurn PD, Miller VM (2000) Neuroprotective effects of female gonadal steroids in reproductively senescent female rats. Stroke 31:161-168.

Alonzi T, Middleton G, Wyatt S, Buchman V, Betz UA, Muller W, Musiani P, Poli V, Davies AM (2001) Role of STAT3 and PI 3-kinase/Akt in mediating the survival actions of cytokines on sensory neurons. Mol Cell Neurosci 18:270-282.

Bjornstrom L, Sjoberg M (2002) Signal transducers and activators of transcription as downstream targets of nongenomic estrogen receptor actions. Mol Endocrinol 16:2202-2214.

Bjornstrom L, Sjoberg M (2005) Mechanisms of estrogen receptor signaling: convergence of genomic and nongenomic actions on target genes. Mol Endocrinol 19:833-842.

Blaskovich MA, Sun J, Cantor A, Turkson J, Jove R, Sebti SM (2003) Discovery of JSI-124 (cucurbitacin I), a selective Janus kinase/signal transducer and activator of transcription 3 signaling pathway inhibitor with potent antitumor activity against human and murine cancer cells in mice. Cancer Res 63:1270-1279.

Bryant DN, Bosch MA, Ronnekleiv OK, Dorsa DM (2005) 17-Beta estradiol rapidly enhances extracellular signal-regulated kinase 2 phosphorylation in the rat brain. Neuroscience 133:343-352.

Bryant DN, Sheldahl LC, Marriott LK, Shapiro RA, Dorsa DM (2006) Multiple pathways transmit neuroprotective effects of gonadal steroids. Endocrine 29:199-207.

Dolcet X, Soler RM, Gould TW, Egea J, Oppenheim RW, Comella JX (2001) Cytokines promote motoneuron survival through the Janus kinasedependent activation of the phosphatidylinositol 3-kinase pathway. Mol Cell Neurosci 18:619-631.

Ehrenreich H, Hasselblatt M, Dembowski C, Cepek L, Lewczuk P, Stiefel M, Rustenbeck HH, Breiter N, Jacob S, Knerlich F, Bohn M, Poser W, Ruther E, Kochen M, Gefeller O, Gleiter C, Wessel TC, De Ryck M, Itri L, Prange $\mathrm{H}$, et al. (2002) Erythropoietin therapy for acute stroke is both safe and beneficial. Mol Med 8:495-505.

Giloh H, Sedat JW (1982) Fluorescence microscopy: reduced photobleaching of rhodamine and fluorescein protein conjugates by n-propyl gallate. Science 217:1252-1255.

Grad JM, Zeng XR, Boise LH (2000) Regulation of Bcl-xL: a little bit of this and a little bit of STAT. Curr Opin Oncol 12:543-549. 
Hattori R, Maulik N, Otani H, Zhu L, Cordis G, Engelman RM, Siddiqui MA, Das DK (2001) Role of STAT3 in ischemic preconditioning. J Mol Cell Cardiol 33:1929-1936.

Jia WW, Wang Y, Qiang D, Tufaro F, Remington R, Cynader M (1996) A bcl-2 expressing viral vector protects cortical neurons from excitotoxicity even when administered several hours after the toxic insult. Brain Res Mol Brain Res 42:350-353.

Justicia C, Gabriel C, Planas AM (2000) Activation of the JAK/STAT pathway following transient focal cerebral ischemia: signaling through Jak1 and Stat3 in astrocytes. Glia 30:253-270.

Komine-Kobayashi M, Zhang N, Liu M, Tanaka R, Hara H, Osaka A, Mochizuki H, Mizuno Y, Urabe T (2006) Neuroprotective effect of recombinant human granulocyte colony-stimulating factor in transient focal ischemia of mice. J Cereb Blood Flow Metab 26:402-413.

Kretz A, Happold CJ, Marticke JK, Isenmann S (2005) Erythropoietin promotes regeneration of adult CNS neurons via Jak2/Stat3 and PI3K/AKT pathway activation. Mol Cell Neurosci 29:569-579.

Kumral A, Genc S, Ozer E, Yilmaz O, Gokmen N, Koroglu TF, Duman N, Genc K, Ozkan H (2006) Erythropoietin downregulates bax and DP5 proapoptotic gene expression in neonatal hypoxic-ischemic brain injury. Biol Neonate 89:205-210.

Levin ER (2002) Cellular functions of plasma membrane estrogen receptors. Steroids 67:471-475.

Levy DE, Darnell Jr JE (2002) Stats: transcriptional control and biological impact. Nat Rev Mol Cell Biol 3:651-662.

Linnik MD, Zahos P, Geschwind MD, Federoff HJ (1995) Expression of bcl-2 from a defective herpes simplex virus- 1 vector limits neuronal death in focal cerebral ischemia. Stroke 26:1670-1674.

Munzberg H, Huo L, Nillni EA, Hollenberg AN, Bjorbaek C (2003) Role of signal transducer and activator of transcription 3 in regulation of hypothalamic proopiomelanocortin gene expression by leptin. Endocrinology 144:2121-2131.

Nefedova Y, Nagaraj S, Rosenbauer A, Muro-Cacho C, Sebti SM, Gabrilovich DI (2005) Regulation of dendritic cell differentiation and antitumor immune response in cancer by pharmacologic-selective inhibition of the janus-activated kinase 2/signal transducers and activators of transcription 3 pathway. Cancer Res 65:9525-9535.

Negoro S, Kunisada K, Tone E, Funamoto M, Oh H, Kishimoto T, YamauchiTakihara K (2000) Activation of JAK/STAT pathway transduces cytoprotective signal in rat acute myocardial infarction. Cardiovasc Res 47:797-805.

O'Lone R, Frith MC, Karlsson EK, Hansen U (2004) Genomic targets of nuclear estrogen receptors. Mol Endocrinol 18:1859-1875.

Parsadanian AS, Cheng Y, Keller-Peck CR, Holtzman DM, Snider WD (1998) Bcl-xL is an antiapoptotic regulator for postnatal CNS neurons. J Neurosci 18:1009-1019.

Qiu J, Bosch MA, Tobias SC, Krust A, Graham SM, Murphy SJ, Korach KS, Chambon P, Scanlan TS, Ronnekleiv OK, Kelly MJ (2006) A G-protein- coupled estrogen receptor is involved in hypothalamic control of energy homeostasis. J Neurosci 26:5649-5655.

Rusa R, Alkayed NJ, Crain BJ, Traystman RJ, Kimes AS, London ED, Klaus JA, Hurn PD (1999) 17Beta-estradiol reduces stroke injury in estrogendeficient female animals. Stroke 30:1665-1670.

Satriotomo I, Bowen KK, Vemuganti R (2006) JAK2 and STAT3 activation contributes to neuronal damage following transient focal cerebral ischemia. J Neurochem 98:1353-1368.

Schweizer U, Gunnersen J, Karch C, Wiese S, Holtmann B, Takeda K, Akira S, Sendtner M (2002) Conditional gene ablation of Stat3 reveals differential signaling requirements for survival of motoneurons during development and after nerve injury in the adult. J Cell Biol 156:287-297.

Solaroglu I, Solaroglu A, Kaptanoglu E, Dede S, Haberal A, Beskonakli E, Kilinc K (2003) Erythropoietin prevents ischemia-reperfusion from inducing oxidative damage in fetal rat brain. Childs Nerv Syst 19:19-22.

Solaroglu I, Cahill J, Jadhav V, Zhang JH (2006a) A novel neuroprotectant granulocyte-colony stimulating factor. Stroke 37:1123-1128.

Solaroglu I, Tsubokawa T, Cahill J, Zhang JH (2006b) Anti-apoptotic effect of granulocyte-colony stimulating factor after focal cerebral ischemia in the rat. Neuroscience 143:965-974.

Sonnenberg JL, Macgregor-Leon PF, Curran T, Morgan JI (1989) Dynamic alterations occur in the levels and composition of transcription factor AP-1 complexes after seizure. Neuron 3:359-365.

Stephanou A, Brar BK, Knight RA, Latchman DS (2000) Opposing actions of STAT- 1 and STAT-3 on the Bcl-2 and Bcl-x promoters. Cell Death Differ 7:329-330.

Suzuki S, Tanaka K, Nogawa S, Dembo T, Kosakai A, Fukuuchi Y (2001) Phosphorylation of signal transducer and activator of transcription-3 (Stat3) after focal cerebral ischemia in rats. Exp Neurol 170:63-71.

Suzuki S, Brown CM, Wise PM (2006) Mechanisms of neuroprotection by estrogen. Endocrine 29:209-215.

Takeuchi J, Shannon W, Aronin N, Schwartz WJ (1993) Compositional changes of AP-1 DNA-binding proteins are regulated by light in a mammalian circadian clock. Neuron 11:825-836.

Wade CB, Dorsa DM (2003) Estrogen activation of cyclic adenosine 5' monophosphate response element-mediated transcription requires the extracellularly regulated kinase/mitogen-activated protein kinase pathway. Endocrinology 144:832-838.

Xu Y, Zhang W, Klaus J, Young J, Koerner I, Sheldahl LC, Hurn PD, Martinez-Murillo F, Alkayed NJ (2006) Role of cocaine- and amphetamine-regulated transcript in estradiol-mediated neuroprotection. Proc Natl Acad Sci USA 103:14489-14494.

Xuan YT, Guo Y, Han H, Zhu Y, Bolli R (2001) An essential role of the JAK-STAT pathway in ischemic preconditioning. Proc Natl Acad Sci USA 98:9050-9055.

Yamashita T, Sawamoto K, Suzuki S, Suzuki N, Adachi K, Kawase T, Mihara M, Ohsugi Y, Abe K, Okano H (2005) Blockade of interleukin-6 signaling aggravates ischemic cerebral damage in mice: possible involvement of Stat3 activation in the protection of neurons. J Neurochem 94:459-468. 